\title{
Study on the Characteristics of a Scintillator for Beta-ray Detection using Epoxy Resin
}

\author{
Jong Soo Nam*, Yong Seok Choi, Sang Bum Hong, Bum Kyung Seo, Jei Kwon Moon, and Jong Won Choi
}

Korea Atomic Energy Research Institute, Decontamination and Decommissioning Technology Development Division, 34057 Daejeon, Republic of Korea

\begin{abstract}
A thin plate of a plastic scintillator for detecting a beta-ray was developed. The plastic scintillator was made using epoxy resin and organic scintillators such as 2.5-diphenyloxazole (PPO) and 1,4-bis [5-phenyl-2-oxazole] benzene (POPOP). The mixture ratio of epoxy resin and the organic scintillators was determined using their absorbance, transmittance, emission spectra, and transparency. Their optimal weight percentage of PPO and POPOP in the organic scintillators was adjusted to 0.2 wt $\%: 0.01 \mathrm{wt} \%$. The prepared plastic scintillator was used to measure the standard source of Sr-90. The pulse height spectra and total counts of the prepared plastic scintillator were similar to a commercial plastic scintillator. Based on the above results, a large-area plastic scintillator was prepared for rapid investigation of a site contaminated with Sr-90. The prepared large-area plastic scintillator was evaluated for the characteristics in the laboratory. The evaluation results are expected to be usefully utilized in the development of a large-area plastic scintillation detector. The large-area plastic scintillation detector developed on the basis of the evaluation results is expected to be utilized to quickly measure the contamination of $\mathrm{Sr}-90$ in the grounds used as a nuclear power facility.
\end{abstract}

\section{Introduction}

The methods for detecting radiation are ionization, excitation, scintillation, extinction and a chemical reaction [1]. Radiation detection using scintillator light produced in a material is one of the oldest and most useful techniques for the detection of a variety of radiation $[2,3]$. Scintillation detectors are widely used for radiation measurements in nuclear and high-energy physics. In addition, new applications in the medical field were recently found [4-6]. A detector using plastic scintillators is well known to have an easy operation because it consists of a chemically stable material [7]. Several studies have been conducted to include the features, such as neutron radiation hardness [8] and sensitivity [9-11], as well as reduced manufacturing costs $[12,13]$. A large-area plastic scintillator of an affordable price has been required by many researchers of nuclear and high-energy physics fields [14-17].

In general, a plastic scintillator using a polymer such as polymethylmethacrylate (PMMA), polyvinyltoluene (PVT) or polystyrene (PS) is added to an organic scintillator. As an organic scintillator, the first solute is p-terphenyl or 2.5-diphenyloxazole (PPO), and the second solute is 1,4-bis [5-phenyl-2-oxazol] benzene (POPOP) [18]. A method for preparing a plastic scintillator is a mixture of a polymer and organic scintillators used for thermal polymerization. Recently, the light generated in the scintillator owing to the external radiation is able to transmit the long distance by combining the optical fiber and the plastic scintillator [19]. However, this method may result in a loss of light in a combination of the optical fiber and the plastic scintillator.

In this study, we prepared a plastic scintillator whose manufacturing process is simple and can be freely shaped. A thin plate of a plastic scintillator was manufactured using epoxy resin as a polymer. The plastic scintillator was made by mixing epoxy resin and organic scintillators under various conditions. We investigated the properties such as the absorbance, transmittance, emission spectra, and transparency of the prepared plastic scintillator. The optimal mixture ratio to prepare the plastic scintillator was derived from the above results. Using the derived results, we made the large-area plastic scintillator, which can quickly measure the contamination site and evaluated characteristics of the large-area plastic scintillator in the laboratory.

\section{Method}

The polymer materials for the preparation of a plastic scintillator were mainly PMMA, PVT or PS. However, PMMA, PVT and PS generate a bad smell in the thermal polymerization process and require a relatively long drying time. A polymer was used as an epoxy resin (WE-300A, Won chemical co. Korea) because no odor

\footnotetext{
Corresponding author: namjs@kaeri.re.kr
} 
resulted from thermal polymerization, and the drying time is short. Organic scintillators were added to the PPO as the first solute and the POPOP as the second solute.
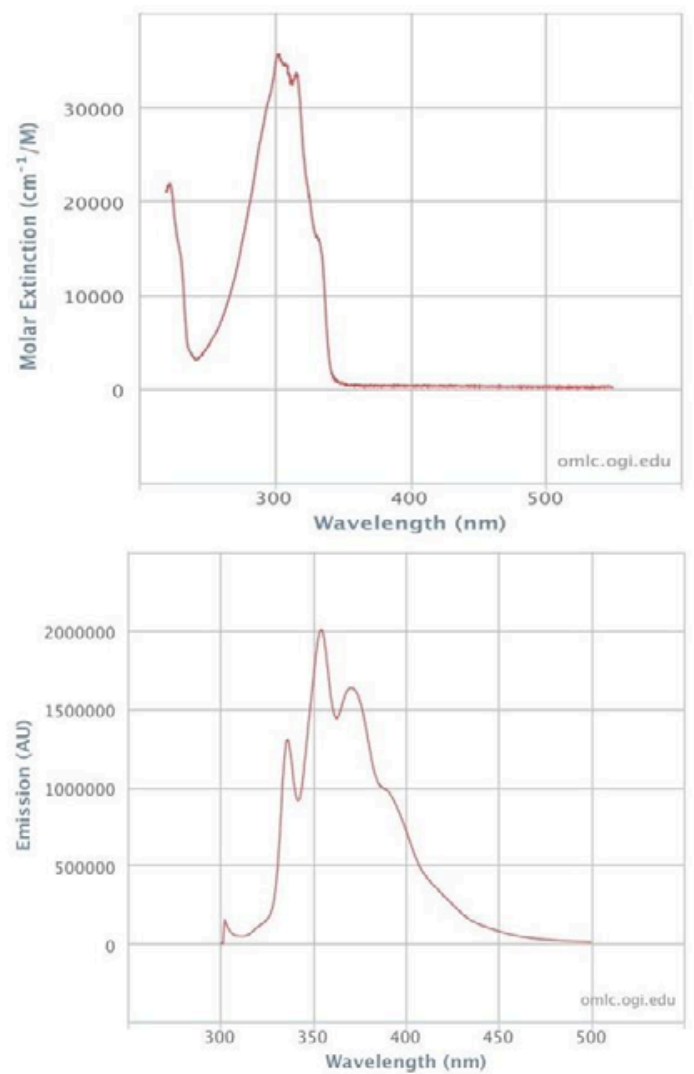

Figure 1. Absorbance (up) and emission (down) spectra of PPO

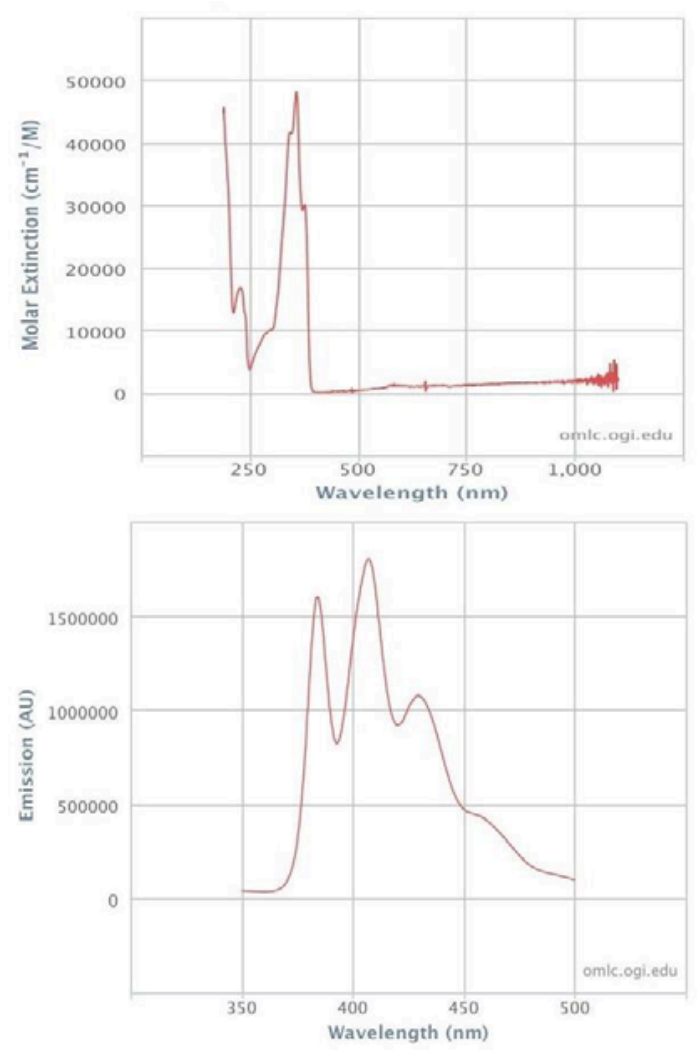

Figure 2. Absorbance (up) and emission (down) spectra of POPOP
Absorbance and emission spectra of PPO and POPOP are shown in Figures 1 and 2 [20, 21]. A polymer material such as an epoxy resin absorbs the energy of the incident radiation. The first solute (PPO) emits scintillation of light in an ultraviolet range (wavelength of 200 to $400 \mathrm{~nm}$ ) by receiving the absorbed energy. However, the wavelength of the ultraviolet range can be easily absorbed in a polymer and is not suitable for the scintillation response of a photomultiplier tube (PMT). The second solute (POPOP) can reduce the absorbance caused in the medium by emitting a blue wave (wavelength of 450 to $490 \mathrm{~nm}$ ) of a long wavelength through the absorbance of scintillation caused from the PPO. In addition, it is a wave shifter for moving the wavelength responsible for the PMT.

The plastic scintillator is made using epoxy resin, PPO and POPOP. The preparation procedure of the plastic scintillator is shown Figure 3. The epoxy resin of a polymer was added in the PPO and POPOP in accordance with the mixture ratio. The scintillation solution into the hardener (WE-300B, Won chemical co. Korea) was mixed by a stirrer for 2 hours at room temperature. After identifying no air bubbles of a polymer solution, the thickness of the plastic scintillator using the polyethylene film to facilitate the removal from the mould was placed in a scintillator volume of approximately 2 to $3 \mathrm{~mm}$. The polymer solution is spread evenly above the polyethylene film. The temperature of the thermal polymerization was successively adjusted at 90 degrees for 12 hours, 110 degrees for 5 hours, and 130 degrees for 2 hours [22].

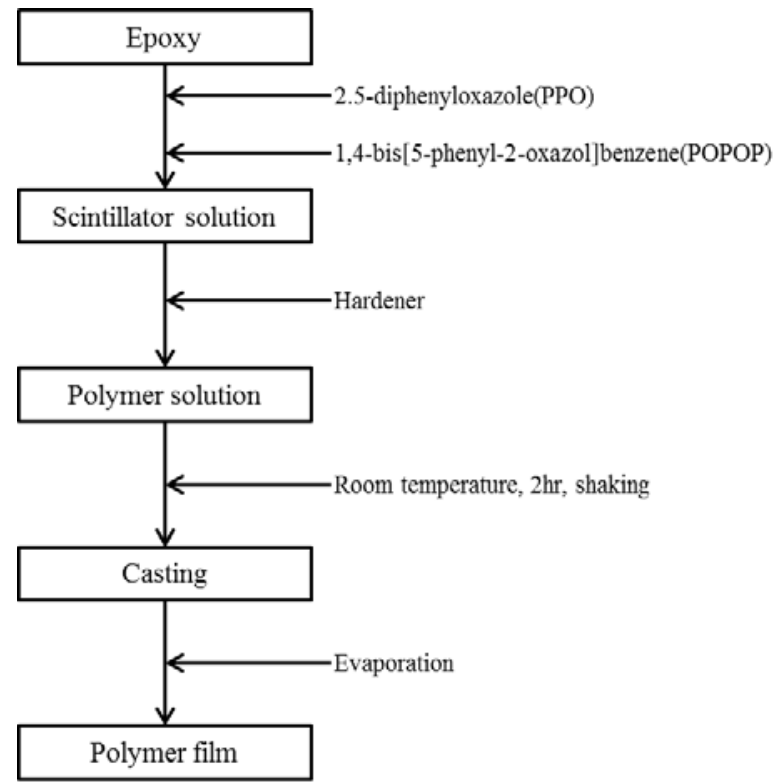

Figure 3. Preparation procedure of the plastic scintillator

The mixture ratio used to make the plastic scintillator is shown in Table 1 . The plastic scintillator made a 50 $\mathrm{mm}$ diameter to evaluate the characteristics according to the mixture ratio. The substance of the plastic scintillator regarding the total weight of epoxy resin was 0.1 to 1 $\mathrm{wt} \%$ and 0.01 to $0.05 \mathrm{wt} \%$ for PPO and POPOP, respectively. The optical properties such as the 
absorbance, transmittance, and emission spectra in the visible light of the prepared plastic scintillator measured using a spectrofluorometer. PMT (H5211A-MOD, Hamamatsu) as a light measuring device was used. The amplification of the scintillation light using PMT was achieved.

Table 1. The mixture ratio of organic scintillators by PPO and POPOP

\begin{tabular}{|c|c|c|c|c|c|}
\hline $\begin{array}{c}\text { Sample } \\
\text { No. }\end{array}$ & $\begin{array}{c}\text { PPO } \\
(\mathrm{wt} \%)\end{array}$ & $\begin{array}{c}\text { POPOP } \\
(\mathrm{wt} \%)\end{array}$ & $\begin{array}{c}\text { Sample } \\
\text { No. }\end{array}$ & $\begin{array}{c}\text { PPO } \\
(\mathrm{wt} \%)\end{array}$ & $\begin{array}{c}\text { POPOP } \\
\text { (wt\%) }\end{array}$ \\
\hline 1 & 0.1 & 0.01 & 7 & 0.1 & 0.0025 \\
\hline 2 & 0.2 & 0.01 & 8 & 0.1 & 0.005 \\
\hline 3 & 0.4 & 0.01 & 9 & 0.1 & 0.01 \\
\hline 4 & 0.6 & 0.01 & 10 & 0.2 & 0.01 \\
\hline 5 & 0.8 & 0.01 & 11 & 0.2 & 0.025 \\
\hline 6 & 1 & 0.01 & 12 & 0.2 & 0.05 \\
\hline
\end{tabular}

\section{Results}

The prepared plastic scintillator was observed with the naked eye. The yellow color phenomenon in the plastic scintillator increased as the amount of PPO occurred. It was founded to be present in the plastic scintillator without melting according to the amount of POPOP. To the naked eye, we could see the transparency of the plastic scintillator by adding the least amount of PPO and POPOP.
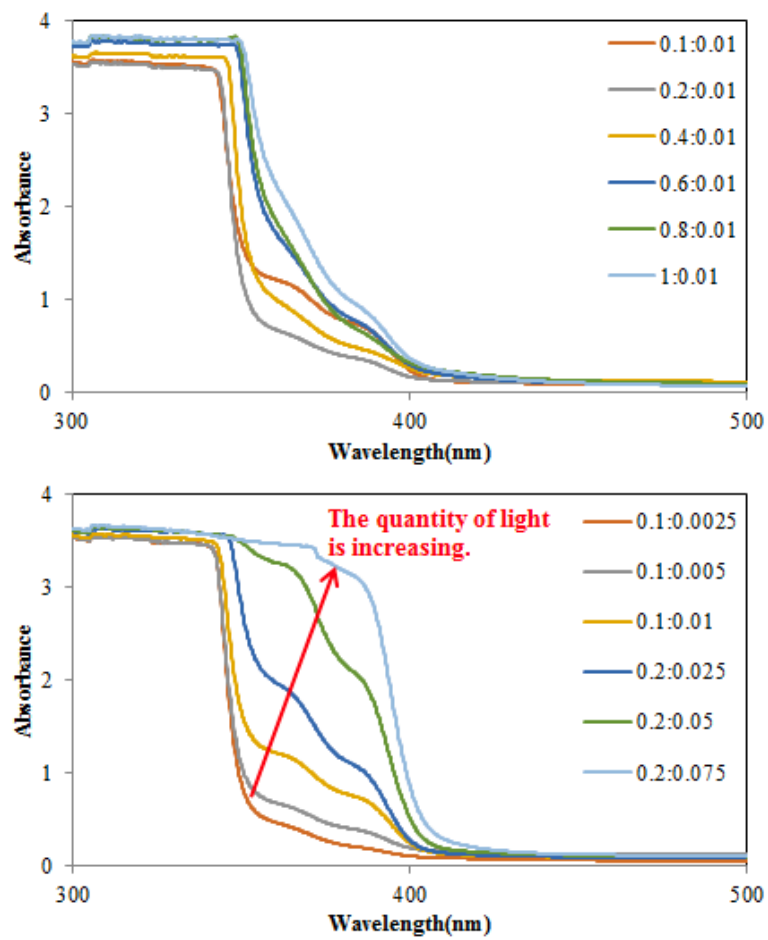

Figure 4. Absorbance spectra according to the amount of PPO (up) and POPOP (down)
The optical properties such as the absorbance, transmittance, and emission spectra in the visible light (wavelength of 380 to $800 \mathrm{~nm}$ ) of the prepared plastic scintillator were measured using a spectrofluorometer.
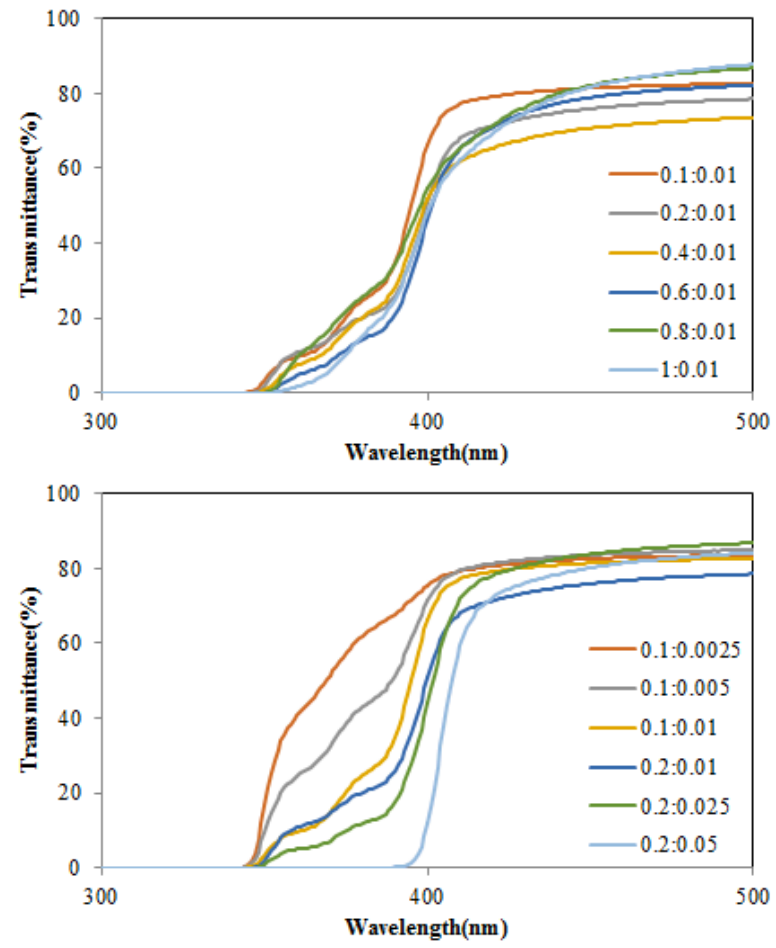

Figure 5. Transmittance spectra according to the amount of PPO (up) and POPOP (down)
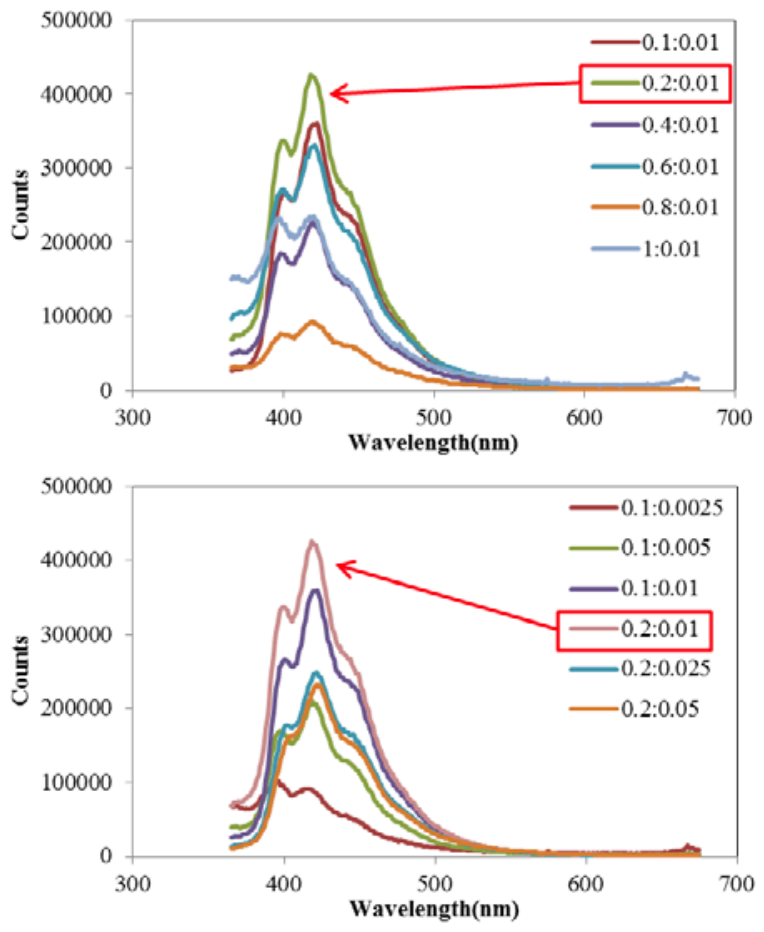

Figure 6. Emission spectra according to the amount of PPO (up) and POPOP (down)

The absorbance, transmittance, and emission spectra were observed to derive the optimal mixture ratio of the plastic scintillator. Figures 4, 5, and 6 show the light 
absorbance, transmittance, and emission spectra according to the amount of PPO and POPOP, respectively. Even if the amount of PPO increases, the absorbance and emission spectra did not show a consistent trend. The transmittance of all plastic scintillators has confirmed to be excellent in the $70 \%$ range. However, if the amount of POPOP increases, the absorbance and emission spectra did show a growing trend. These results indicate that the amount of PPO is independent, and the greater the amount of increase in POPOP, the more the quantity of light that is increasing. However, the plastic scintillator increased as the amount of PPO was remarkably changed to yellow. In addition, there is a problem without melting to POPOP.

Based on the above measurement result, the best plastic scintillator is slightly yellowing, and excellent in absorbance and transmittance, and its amount of emission of light is the best. The weight percentage of PPO and POPOP in an organic scintillator was adjusted to $0.2 \mathrm{wt} \%: 0.01 \mathrm{wt} \%$.

The detection efficiency of a plastic scintillator was evaluated based on the content prepared to the optimal mixture ratio. A plastic scintillator was optically connected to the PMT with optical grease and wrapped with a black tape after placing a white paper as a reflector [23]. Figure 7 shows the plastic scintillator measurement setup. The measurement system was placed in a light shield box before loading in the darkroom. The distance of the plastic scintillator and standard source was maintained at $20 \mathrm{~mm}$.

The standard source is $\mathrm{Sr}-90$ with a radioactivity of $0.1 \mu \mathrm{Ci}$. Figure 8 shows the decay scheme of $\mathrm{Sr} / \mathrm{Y}-90$, which emits beta-rays without emitting a gamma-ray. The contamination of Sr-90 is measured to have a highenergy beta radiation of $2.3 \mathrm{MeV}$ emitted by beta decay of Y-90.

Figure 9 shows the spectra of the commercial and prepared plastic scintillator. As shown in the figure, the pulse height spectra and total counts of the prepared plastic scintillator were similar to a commercial plastic scintillator.

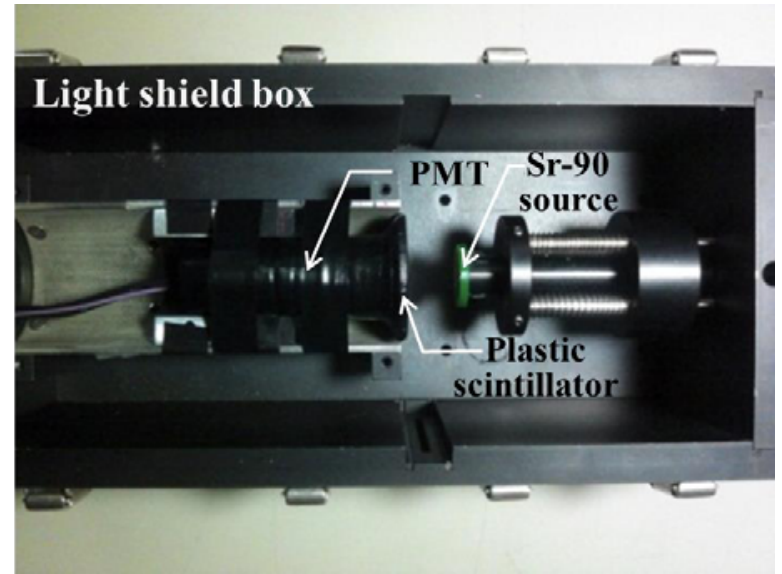

Figure 7. The plastic scintillator measurement setup

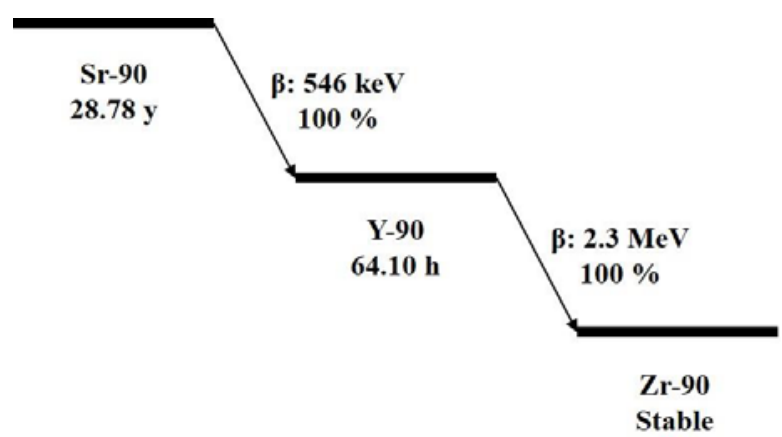

Figure 8. Decay scheme of Sr-90
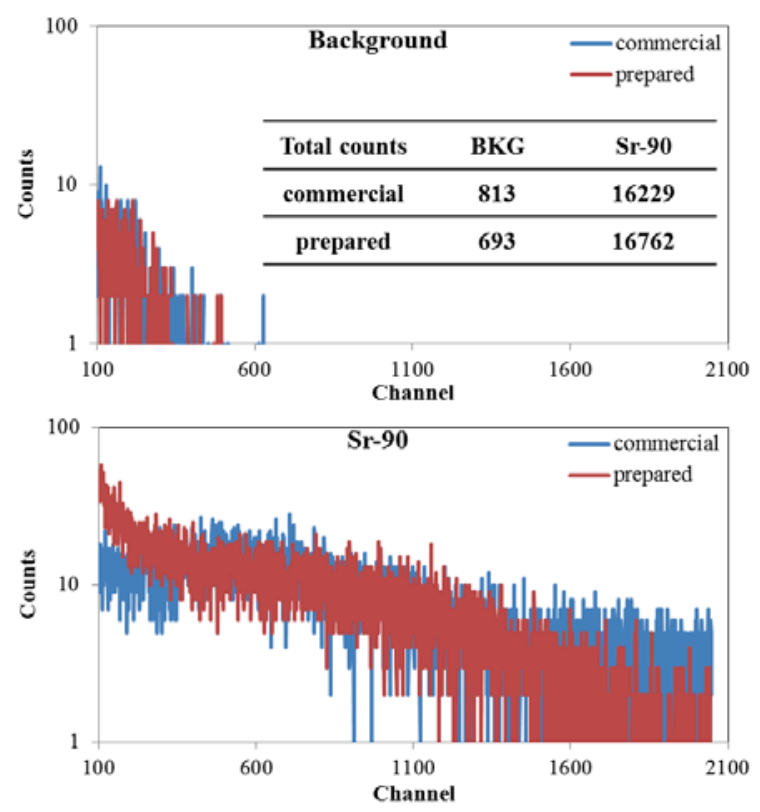

Figure 9. Radiation spectra of the plastic scintillator

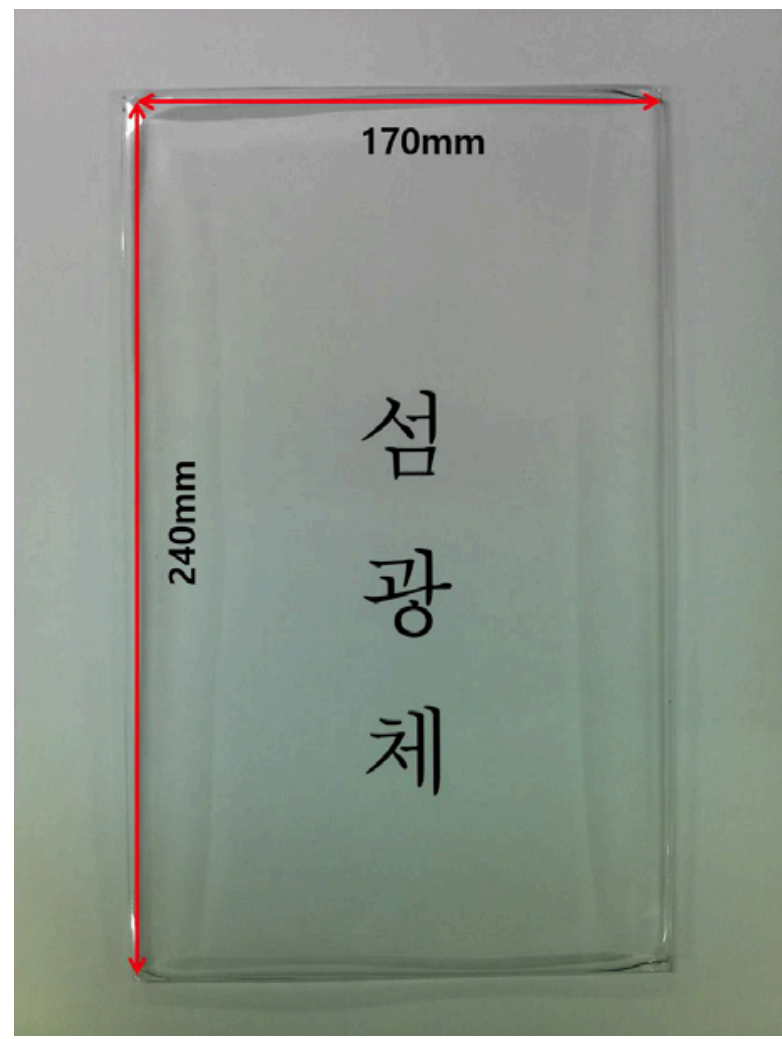

Figure 10. The large-area plastic scintillator 
Based on the characteristic results, a large-area plastic scintillator was prepared. The size of the largearea plastic scintillator was similar to the window size of a typical pancake-type $\alpha \beta$ surface contamination counter. The size of the large-area plastic scintillator is $240 \mathrm{~mm}$ (length) x $170 \mathrm{~mm}$ (width) x $3 \mathrm{~mm}$ (thickness) (Figure 10). In order to make the large-area plastic scintillator, it was produced above a metal mould of the same size. Polyethylene film was used as a releasing agent to ease the removal of the large-area plastic scintillator from the metal mould. The large-area plastic scintillator was pressed by a metal lid to prevent twisting.

We wanted to evaluate the characteristics of the large-area plastic scintillator. It was difficult to connect them because of the difference in size between the largearea plastic scintillator and PMT.

\section{Conclusion}

A thin plate of a plastic scintillator with a simple preparation process can be freely shaped using epoxy resin and organic scintillators such as PPO and POPOP. PPO emits a scintillation of light in the ultraviolet range, and POPOP is a wave shifter for moving the wavelength responsible for the PMT. When preparing the plastic scintillator, the temperature of the thermal polymerization rose sequentially. Otherwise, a thermal polymerization at high temperature (for example 130 degrees for 2 hours) was crushed, or at too low a temperature (for example, less than 80 degrees) was weak to be easily broken down. The mixture ratio of PPO and POPOP was determined using their absorbance, trasmittance, emission spectra, and transparency. The optimal weight percentage of PPO and POPOP in an organic scintillator was determined to be $0.2 \mathrm{wt} \%: 0.01$ $\mathrm{wt} \%$. The pulse height spectra of a plastic scintillator were evaluated based on the content prepared to the optimal mixture ratio, and the prepared plastic scintillator was similar to a commercial plastic scintillator.

Based on the above results, the large-area plastic scintillator of the window size of a typical pancake-type $\alpha \beta$ surface contamination counter was prepared. We want to evaluate the characteristics of the large-area plastic scintillator. However, there were the difficulties in evaluating the characteristics of the large-area plastic scintillator. The cross-sectional area of the large-area plastic scintillator is significantly different to PMT. The tool will be necessary to connect the large-area plastic scintillator and PMT. The tool for connecting them generally utilizes a light guide. The material of the light guide is used in a highly transparent acrylic. Acrylic of a pyramid shape to connect them will be made. Then, a characteristic evaluation of the large-area plastic scintillator needs to be carried out. In addition, the light guide serves to enhance the focusing efficiency of the light.
It will be utilized for the preparation of large-area plastic scintillators for a rapid investigation of the decommissioning site of nuclear power plants contaminated with Sr-90.

\section{Acknowledgement}

This work has been carried out under the long-term nuclear research and development program (2012M2A8A5025996) sponsored by Ministry of Science, ICT and Future Planning of Korea.

\section{References}

1. J.H.Kim, K.S.Joo, Journal of IKEEE 18, 456 (2014)

2. G.F.Knoll, Radiation Detection \& Measurement, 219 (2010)

3. S.N.Ahmed, Physics \& Engineering of Radiation Detection, 319 (2007)

4. T.K.Lewellen, Phy Med Biol 53, R287 (2008)

5. L.Archambault, T.M. Briere, F.Pönish, L.Beaulieu, D.A.Kuban, A.Lee, S.Beddar, Int J Radiat Oncol 78, 280 (2010)

6. A.Vandenbroucke, A.M.K.Foudray, P.D.Olcott, C.S.Levin, Phy Med Biol 55, 5895 (2010)

7. M.Watanabe, M.Katsumata, H.Ono, T.Suzuki, H.Miyata, Y.Itoh, K.Ishiba, M.Tamura, Y.Yamaguchi, Nucl Instrum Meth A 770, 197 (2015)

8. A.Quaranta, S.Carturan, T.Marchi, M.Cinausero, C.Scian, V.L.Kravchuk, M.Degerlier, F.Gramegna, M.Poggi, G.Maggioni, Opt Mater 32, 1317 (2010)

9. V.B.Brudanin, V.I.Bregadze, N.A.Gundorin, D.V.Filossofov, O.I.Kochetov, I.B.Nemtchenok, A.A.Smolnikov, S.I.Vasiliev, Pariticles and Nuclei Letters 109, 69(2001)

10. L.Ovechkina, K.Riley, S.Miller, Z.Bell, V.Nagarkar, Physics Procedia 2, 161 (2009)

11. A.Quaranta, S.Carturan, T.Marchi, M.Buffa, M.Degerlier, M.Cinausero, G.Guastalla, F.Gramegna, G.Valotto, G.Maggioni, V.L.Kravchuk, J Non-Cryst Solids 357, 1921 (2011)

12. A.Pla-Dalmau, A.D.Bross, K.L.Mellott, Nucl Instrum Meth A 466, 482 (2001)

13. H.Nakamura, Y.Shirakawa, S.Takahashi, H.Shimizu, Europhysics Letters 95, 22001 (2011)

14. The CMS collaboration, The Compact Muon Solenoid Technical Proposal, CERN/LHCC 94-38 (1994)

15. The CDF $\amalg$ collaboration, The CDF- II Detector Technical Design Report, Fermilab-Pub-96/390-E (1996)

16. A.L.C.Sanchez, H.Miyata, N.Nakajima, H.Ono, Y.Fujii, S.Itoh, F.Kajino, J.Kanzaki, K.Kawagoe, S.Kim, S.Kishimoto, T.Matsumoto, H.Matsunaga, A.Nagano, R.Nakamura, K.Sekiguchi, T.Takeshita, N.Uchida, Y.Yamada, S.Yamamoto, S.Yamauchi, Nucl Instrum Meth A 546, 535 (2005)

17. H.Ono, H.Miyata, S.Iba, N.Nakajima, A.L.C.Sanchez, Y.Fujii, S.Itoh, F.Kajino, J.Kanzaki, K.Kawagoe, 
S.Kim, S.Kishimoto, T.Matsumoto, H.Matsunaga, A.Nagano, R.Nakamura, K.Sekiguchi, T.Takeshita, Y.Tamura, S.Yamauchi, Nucl Instrum Meth A 600, 398 (2009)

18. B.K.Seo, G.H.Kim, Z.H.Woo, W.J.Oh, K.W.Lee, M.J.Han, Analytical Science \& Technology 18, 495 (2005)

19. C.H.Park, J.H.Moon, B.K.Seo, Radiat Meas 46, 687 (2011)

20. http://omlc.org/spectra/PhotochemCAD/html/0.20.h tml

21. http://omlc.org/spectra/PhotochemCAD/html/0.77.h tml

22. J.S.Nam, Y.S.Choi, S.B.Hong, B.K.Seo, J.K.Moon, J.W.Choi, Abstracts of Proceedings of the Korean Radioactive Waste Society AUTUMN 2015, 13, 287 (2015)

23. E.P.Jacosalem, S.Iba, N.Nakajima, H.Ono, A.L.C.Sanchez, A.M.Bacala, H.Miyata, GLD Calorimeter Group, Pramana 69, 1051 (2007) 